\title{
NOREPINEFRINA O ETILEFRINA EN TRATAMIENTO DE HIPOTENSIÓN ARTERIAL INTRAOPERATORIA PARA ARTROPLASTIA TOTAL DE CADERA
}

\author{
NOREPINEPHRINE OR ETILEPHRINE IN THE TRATAMIENT OF \\ INTRAOPERATIVE ARTERIAL HYPOTENSION FOR TOTAL HIP \\ ARTHROPLASTY
}

\author{
Patricia Alegre Andrade ${ }^{1}$, Alexander Arnica Gamón²
}

\section{RESUMEN}

La complicación frecuente, posterior a anestesia raquídea en artroplastia de cadera es Hipotensión Arterial Intraoperatoria, por eso la necesidad de evaluar un vasopresor que es útil para este propósito.

Objetivos: Evaluar uso de norepinefrina y etilefrina para prevención de la hipotensión intraoperatoria inducida por la anestesia espinal en pacientes sometidos a artroplastia total de cadera.

Materiales y Métodos: Ensayo clínico, simple ciego, transversal y prospectivo. 38 pacientes ambos sexos, edad 60 a 85 años, ASA II-III, anestesia espinal y acepten participar del estudio. Exclusión a edad $<60$ y $>85$ años, ASA IV-V, anestesia general, HTA y cardiopatía descontrolada. Grupo de estudio: grupo E (Etilefrina $2 \mathrm{mg}$ bolo IV) y grupo $\mathrm{N}$ (Norepinefrina infusión 0.03 a $0.05 \mathrm{mcg} / \mathrm{kg} / \mathrm{min} \mathrm{IV).} \mathrm{El} \mathrm{análisis} \mathrm{estadístico} \mathrm{se} \mathrm{realizó} \mathrm{prueba} \mathrm{T} \mathrm{student.}$

Resultados: Edad media grupo E: 68 años y el grupo N 69,94 años. Presión arterial media basal (grupo E): 74,3 $\mathrm{mmHg}$ y (Grupo N): 72,4 mmHg, se encontró significancia estadística valor $\mathrm{p}<0,05$ posterior a administración de anestésicos locales. Por su parte, la frecuencia cardiaca basal es de 73 latidos (grupo E), posterior a administración de etilefrina: 95 latidos; Grupo N: 70 basal y descenso de 10 latidos posterior a infusión de norepinefrina. Con una significancia estadística (valor $\mathrm{p}<0,05$ ) en los tres momentos de medición (frecuencia cardiaca modificada con vasopresor, frecuencia cardiaca durante la cirugía y al finalizar la cirugía).

Conclusiones: Uso de norepinefrina en infusión mantiene estabilidad hemodinámica dentro rangos normales. No hubo incidentes del uso de vasopresores por vía periférica.

\section{ABSTRACT}

The frequent complication, after spinal anesthesia in hip arthroplasty is Intraoperative Arterial Hypotension, so the need to evaluate a vasopressor that is useful for this purpose.

Objectives:To evaluate the use of norepinephrine and etilefrine for the prevention for intraoperative hypotension induced by spinal anesthesia in patients undergoing total hip arthroplasty.

Methods: Clinical trial, simple blind, transverse and prospective. 38 patients of both sex, age 60 to 85 years, ASA II-III, spinal anesthesia and accept to participate in the study. Exclusion criteria: age $<60$ and $>85$ years, ASA IV$\mathrm{V}$, general anesthesia, hypertension and uncontrolled heart disease. Assigned in 2 groups: group $\mathrm{E}$ (Etilefrine 2 $\mathrm{mg}$ bolus IV) and group N (Norepinephrine infusion 0.03 to $0.05 \mathrm{mcg} / \mathrm{kg} / \mathrm{min}$ IV). The statistical analysis was performed student T.

Results:Age in group E: 68 years and group N 69.94 years. Mean arterial pressure baseline (group E): $74.3 \mathrm{mmHg}$, y (group N): $72.4 \mathrm{mmHg}$, Statistical significance $p$ value $<0.05$ after administration of local anesthestics. In group $E$, the baseline heart rate is 73 beats when using ethylephrine up to 95 beats. Group N: baseline of 70 and descent of 10 beats when the norepinephrine infusion is started. The Statistical significance ( $P$ value $<0.05)$ in three moment (heart rate with vassopresor, durant surgery and end surgery).

Conclusions: Use of norepinephrine in infusion maintains hemodynamic stability within normal ranges. There were no incidents of peripheral vasopressor use.

\begin{tabular}{|c|}
\hline iología. \\
\hline $\begin{array}{l}\text { Correspondencia a: } \\
\text { Nombre: Patricia Alegr } \\
\text { Andrade } \\
\text { Teléfono: + (591) } 72292297 \\
\text { Email:wonderful122.paa } \\
\text { gmail.com }\end{array}$ \\
\hline
\end{tabular}

Palabras clave: Norepinefrina, Etilefrina, hipotensión, intraoperatoria.

Keywords: Norepinephrine, Etilefrine, intraoperative, hypotension.

Procedencia y arbitraje: no comisionado, sometido a arbitraje externo.

Recibido para publicación: 15 de septiembre de 2019 Aceptado para publicación: 6 de enero de 2020

Citar como:

Alegre $\mathrm{P}$, Arnica A Norepinefrina o etilefrina en tratamiento de hipotensión arterial intraoperatoria para artroplastía total de cadera. Rev Cient Cienc Med 2019;22(2):

29-35 


\section{INTRODUCCIÓN}

T a complicación intraoperatoria más frecuente Ues la Hipotensión Arterial Intraoperatoria (HIO) en un $87 \%$ en pacientes sometidos a artroplastia total de cadera bajo bloqueo neuroaxial ${ }^{1}$.

La Artroplastia Total Primaria de Cadera (ATPC) es un procedimiento ortopédico común en la práctica clínica consistente en reemplazar el componente femoral y acetabular de la articulación de la cadera, alterados por diversas patologías ${ }^{2}$.

El tratamiento quirúrgico de la fractura de cadera lleva a mayor tiempo de estadía en el hospital con una reinserción a sus actividades diarias de forma paulatina ${ }^{3}$.

Las principales comorbilidades son enfermedad cardiovascular (35\%), enfermedad respiratoria (14\%), enfermedad cerebrovascular (13\%), diabetes $(9 \%)$, cáncer $(8 \%)$ y enfermedad renal bajo tratamiento $(3 \%)^{4}$.

Se describen varias técnicas anestésicas para realizar la cirugía de cadera, entre los cuales, desde la anestesia general, neuroaxial, mas adición de bloqueo nervioso periférico continuo y por último, infiltración local. El tipo de anestesia depende de varios factores y comorbilidades que presente el paciente geriatra. Sin embargo, se debe realizar énfasis en la analgesia postoperatoria ${ }^{5}$.

El uso de anestesia neuroaxial ha sido asociado con riesgo de bradicardia y asistolia, así como de hipotensión ${ }^{4}$.

Sin embargo, hay que evitar los episodios de hipotensión excesiva (presión arterial sistólica < $100 \mathrm{mmHg}$, disminución de más del $30 \%$ de la presión arterial preoperatoria) y prolongada $(>5-10$ minutos $)^{6}$.

La piedra angular del tratamiento de la $\mathrm{HIO}$ es el uso de fármacos vasopresores. El vasopresor ideal para el tratamiento de la $\mathrm{HIO}$, debe tener un bajo costo, estar disponible rápidamente, tener un rápido inicio de acción, ser seguro y tener efectos benéficos para el paciente ${ }^{7}$.

La norepinefrina es una amina simpaticomimética potente vasoconstrictora e inotrópica positiva. $\mathrm{Su}$ acción directa sobre todo en los receptores adrenérgicos alfa y en menor proporción en los adrenérgicos beta-2. La elevación de la presión arterial origina una respuesta vagal refleja compensatoria que induce la disminución de la frecuencia cardiaca ${ }^{8}$.

La Etilefrina es un agente simpaticomimético de acción directa con elevada afinidad para los receptores alfa- 1 , beta- 1 y beta- 2 . Por este motivo es capaz de potenciar la contractibilidad cardiaca y aumentar el gasto cardiaco elevando el volumen sistólico ${ }^{9}$, la etilefrina es el vasopresor más usado en Bolivia ${ }^{10}$.

El objetivo del estudio es evaluar el uso de norepinefrina y etilefrina en prevención de la hipotensión intraoperatoria inducida por la anestesia espinal en pacientes sometidos a artroplastia total de cadera en el departamento de Anestesiología del Hospital Obrero N ${ }^{\circ}$ “Caja Nacional de Salud”.

\section{MATERIALES Y MÉTODOS}

Es un ensayo clínico, simple ciego, transversal y prospectivo. El presente estudio se realizó en el periodo del 2018 en el Hospital Obrero $\mathrm{N}^{\circ} 2$.

La población total de 70 pacientes programados para prótesis de cadera, la muestra estuvo conformada de 19 pacientes por grupo, con una significancia de $(\alpha=0.05)$ y poder $(\beta=0.20)$, sometidos a prótesis de cadera en base a los criterios de inclusión: paciente de ambos sexos, edad comprendida entre 60 a 85 años, Clasificación del Estado Físico ASA II-III, anestesia regional espinal y acepten participar del estudio. Criterios de exclusión: edad menor de 60 y mayor de 85 años, ASA IV- V, anestesia general, hipertensión y cardiopatía no controlada, deterioro cognitivo, rechacen participar en el estudio.

Los participantes fueron asignados en dos grupos: grupo E (Etilefrina) y grupo N (Norepinefrina).

La preparación de los vasopresores se realizó de la siguiente manera:

Grupo E: Etilefrina clorhidrato una ampolla de $10 \mathrm{mg}$ diluida con $9 \mathrm{ml}$ de agua destilada o solución fisiológica $0.9 \%$ (concentración $1 \mathrm{mg} / \mathrm{ml}$ ).

Grupo N: Noradrenalina para infusión: se diluyeron una ampolla de $4 \mathrm{mg}(4 \mathrm{ml})$ de Noradrenalina en $46 \mathrm{ml}$ de solución fisiológica en jeringa de $50 \mathrm{ml}$ obteniendo una concentración de $0,08 \mathrm{mg} / \mathrm{ml}$.

Técnica:

Pacientes ingresan a quirófano con dos catéteres venosos periférico de calibre \#18, el sitio de punción intravenosa es más proximal posible en miembro superior (antecubital, cefálica, basílica); evitando áreas de pliegues cutáneos, asegurando su correcta permeabilidad y funcionamiento.

Ambos grupos se realizó bloqueo espinal en espacio lumbar L3 - L4 o L4 -L5 previa asepsia y antisepsia de región a puncionar con aguja Whitacre punta de lápiz $\mathrm{N}^{\circ} 27$ o 25, se administró $15 \mathrm{mg}$ de Bupivacaina hiperbárica al 0,5\% mas Fentanyl $20 \mathrm{mcg}$.

Se monitorizo frecuencia cardiaca, presión arterial no invasiva, oximetría de pulso, electrocardiograma antes de iniciar procedimiento anestésico y luego cada 5 minutos posterior a la administración de los 
anestésicos locales. Se coloca puntas nasales a 2 litros por minuto, administración de líquidos cristaloides a razón de $15 \mathrm{ml} / \mathrm{kg}$ por $8-10 \mathrm{ml} / \mathrm{kg}$.

Con respecto, a la bradicardia se define frecuencia cardiaca por debajo de 45 latidos por minuto. Y fue tratado con $0.5 \mathrm{mg}$ de atropina de forma intravenosa.

Así mismo, para el tratamiento de la Hipotensión, la hipotensión en los pacientes fue definido por un descenso de la presión arterial sistólica debajo de 100 mmhg o del $20 \%$ del basal para que posteriormente reciban el tratamiento con los medicamentos como etilefrina o norepinefrina.

Se administró Etilefrina $2 \mathrm{mg}$ en bolo las veces necesarias para tratar episodios de hipotensión intraoperatoria en el grupo $\mathrm{E}$ y se inició infusión de norepinefrina durante la cirugía a una dosis de 0.03 a $0.05 \mathrm{mcg} / \mathrm{kg} / \mathrm{min}$. Se suspende en caso de presentar efectos adversos al medicamento y se retira del estudio.

Análisis Estadístico

Se estimó 19 pacientes por grupo con una significancia de $(\alpha=0.05)$ y poder $(\beta=0.20)$. El análisis estadístico para las variables continuas se realizó la media y la desviación estándar y en el caso de las variables nominales se calculó sus frecuencias y porcentajes. Se aplicó prueba de T de Student, se consideraron estadísticamente significativos valores menores a 0,05. El análisis estadístico se realizó en

\section{Gráfico 1. GENERO DE AMBOS GRUPOS}

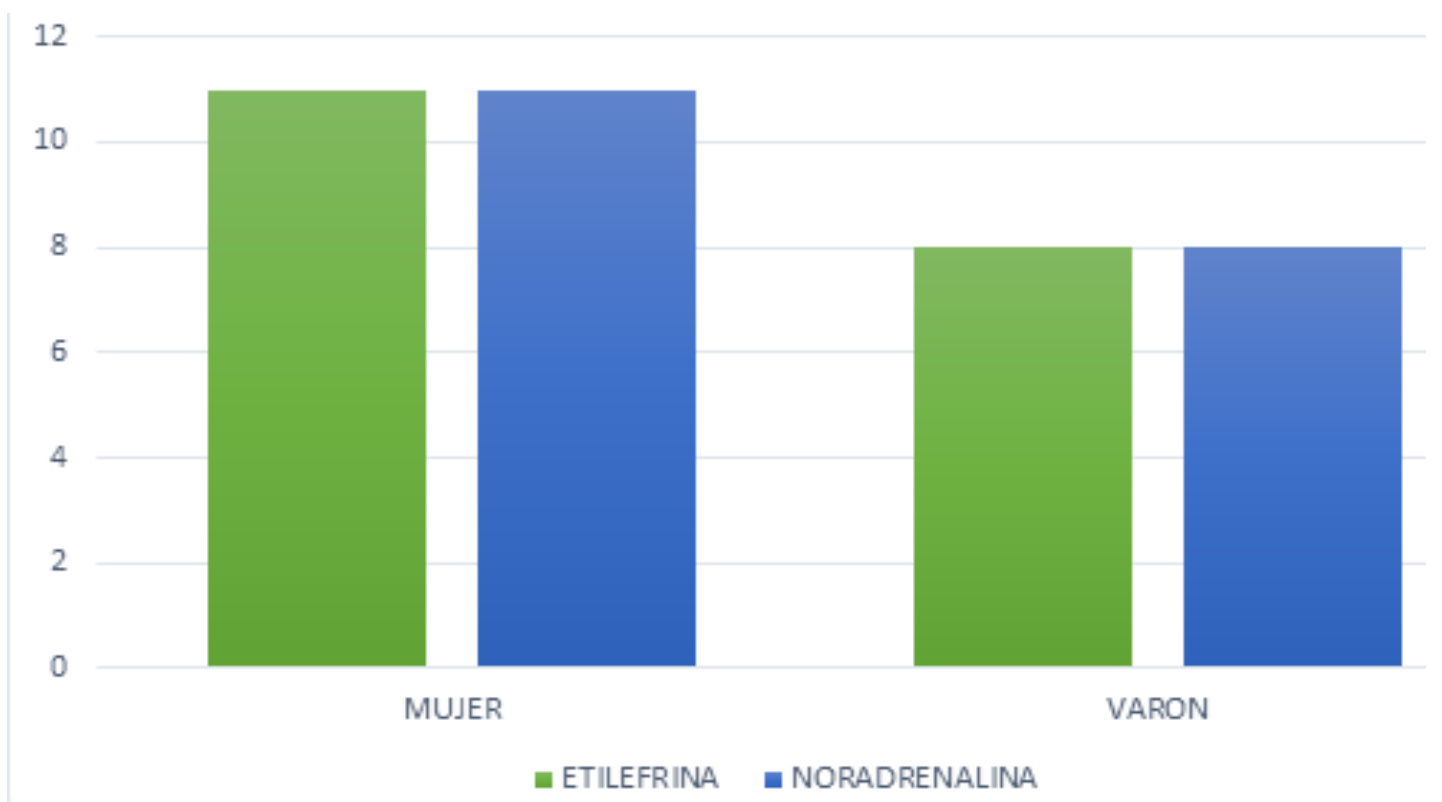

Gráfico 1. En cuanto al género, se trató de tener el mismo número de pacientes: 8 varones y 11 mujeres en ambos grupos de estudio.

Fuente de Elaboración Propia.
SSPS versión 22.0 para Windows y la tabulación de datos en Microsoft Excel 2010.

Consideraciones éticas

Los autores declaran que no existe conflicto de interés alguno. Para realizar el estudio se envió, el protocolo del trabajo al jefe de Departamento de Anestesiología y además, la obtención del consentimiento informado por parte de los pacientes, para poder realizar el estudio.

\section{RESULTADOS}

Las características de la población de estudio demuestran que según:

La edad media en el grupo Etilefrina es de 68 años $\pm 7,6$ años. En el grupo Norepinefrina, la edad media $69,94 \pm 6,6$ años.

En cuanto al género no hay ninguna diferencia significativa entre ambos grupos. Ver Gráfico 1 .

Con respecto al diagnóstico operatorio de los pacientes, en ambos grupos como de etilefrina $y$ noradrenalina se distribuyen con más frecuencia para el diagnóstico de fractura transtrocanterica cadera con un $79 \%$ y $89,4 \%$ respectivamente; y con menor frecuencia para el diagnóstico de coxartrosis 5,3\% en ambos grupos. Grafico 2 .

Se tomaron en cuenta las presiones arteriales medias (PAM), en el grupo de etilefrina: la presión arterial media es de $74,3 \mathrm{mmHg}$, antes de administrar 
de anestésicos locales y durante la administración de anestésicos locales es de 72,2 $\mathrm{mmHg}$, posterior a la administración de los anestésicos locales se encontró un descenso marcado de $58 \mathrm{mmHg}$, cuando se administra etilefrina sube hasta $66,1 \mathrm{mmHg}$ y se mantiene entre 69,1 a 70, $1 \mathrm{mmHg}$.

En el grupo de norepinefrina, la presión arterial media se observa una media basal de $72,4 \mathrm{mmHg}$,

\section{Grafico 2. DIAGNOSTICO PREOPERATORIO}

\section{DIAGNOSTICO PREOPERATORIO}

fractura transtrocantérica cadera
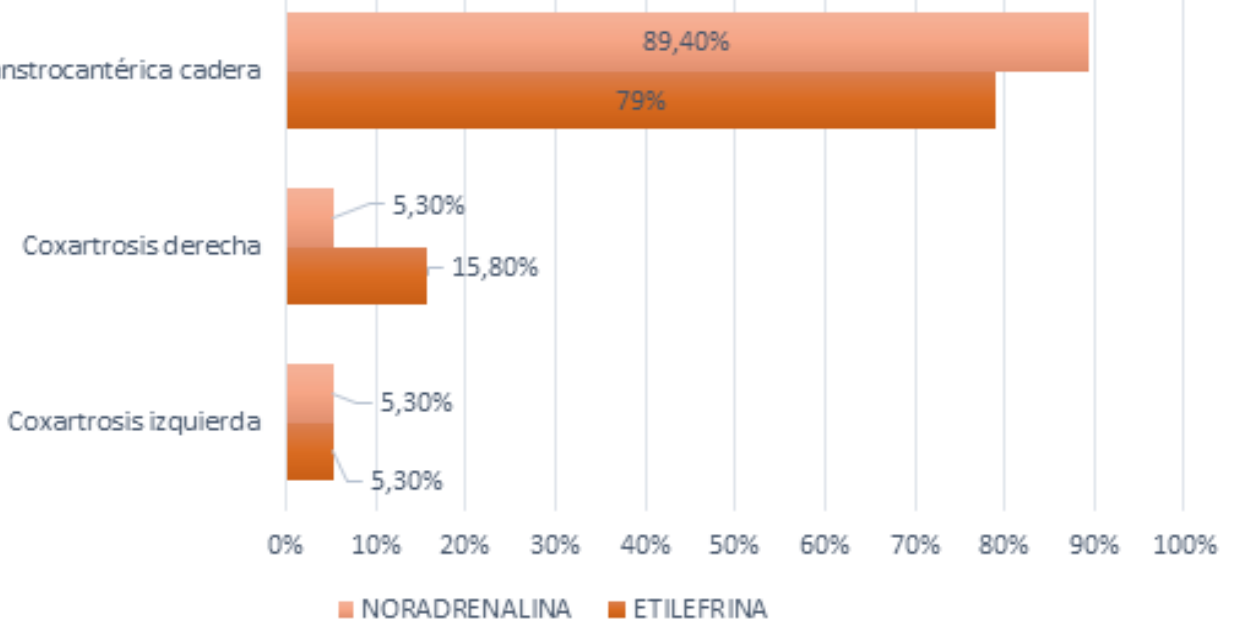

Grafico 2. El diagnóstico más frecuente es la fractura transtrocanterica de cadera.

Fuente de Elaboración Propia.

Grafico 3. PRESION ARTERIAL MEDIA

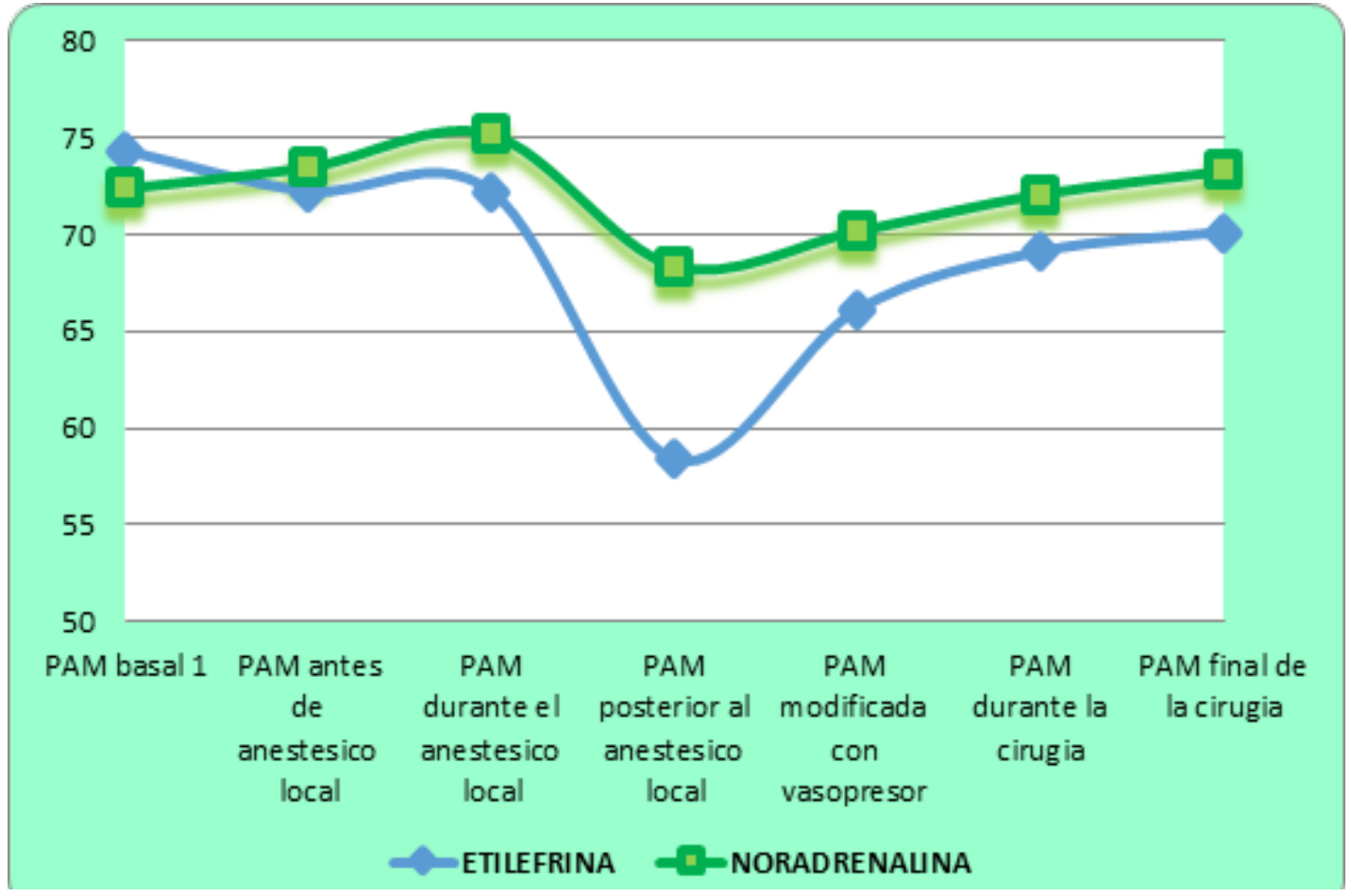

Grafico 3. La presión arterial media con la administración de norepinefrina mantiene en mejores condiciones.

Fuente de Elaboración Propia. 
posterior a la administración de anestésicos locales se observa un descenso de $6 \mathrm{mmHg}$ y durante todo el procedimiento anestésico - quirúrgico se mantiene entre 73,5 a 73,3 mmHg. Ver Gráfico 3. Al realizar el análisis estadístico se encontró que el cambio significativo entre ambos grupos fue posterior a la administración de los anestésicos locales con una significancia estadística valor $\mathrm{p}<0,05$.

El análisis de la frecuencia cardiaca media basal en el grupo E es de 73 latidos por minuto y 70 latidos por minuto en el grupo N. Se puede observar que posterior a la administración de etilefrina asciende hasta 95 latidos por minuto y se mantiene hasta el final mientras que en el grupo de norepinefrina basal de 70 y se observa un descenso de 10 latidos por minuto, todo lo contrario al usar etilefrina. Con una significancia estadística (valor $\mathrm{p}<0,05$ ) en los tres momentos de medición (frecuencia cardiaca modificada con vasopresor, frecuencia cardiaca durante la cirugía y al finalizar la cirugía) Grafico 4.

La saturación de oxigeno se mantuvo entre 94 a 93 $\%$ en ambos grupos.

El efecto secundario que se observó descenso en la frecuencia cardiaca pero sin repercusión en el grupo de norepinefrina.

\section{Grafico 4. FRECUENCIA CARDIACA}

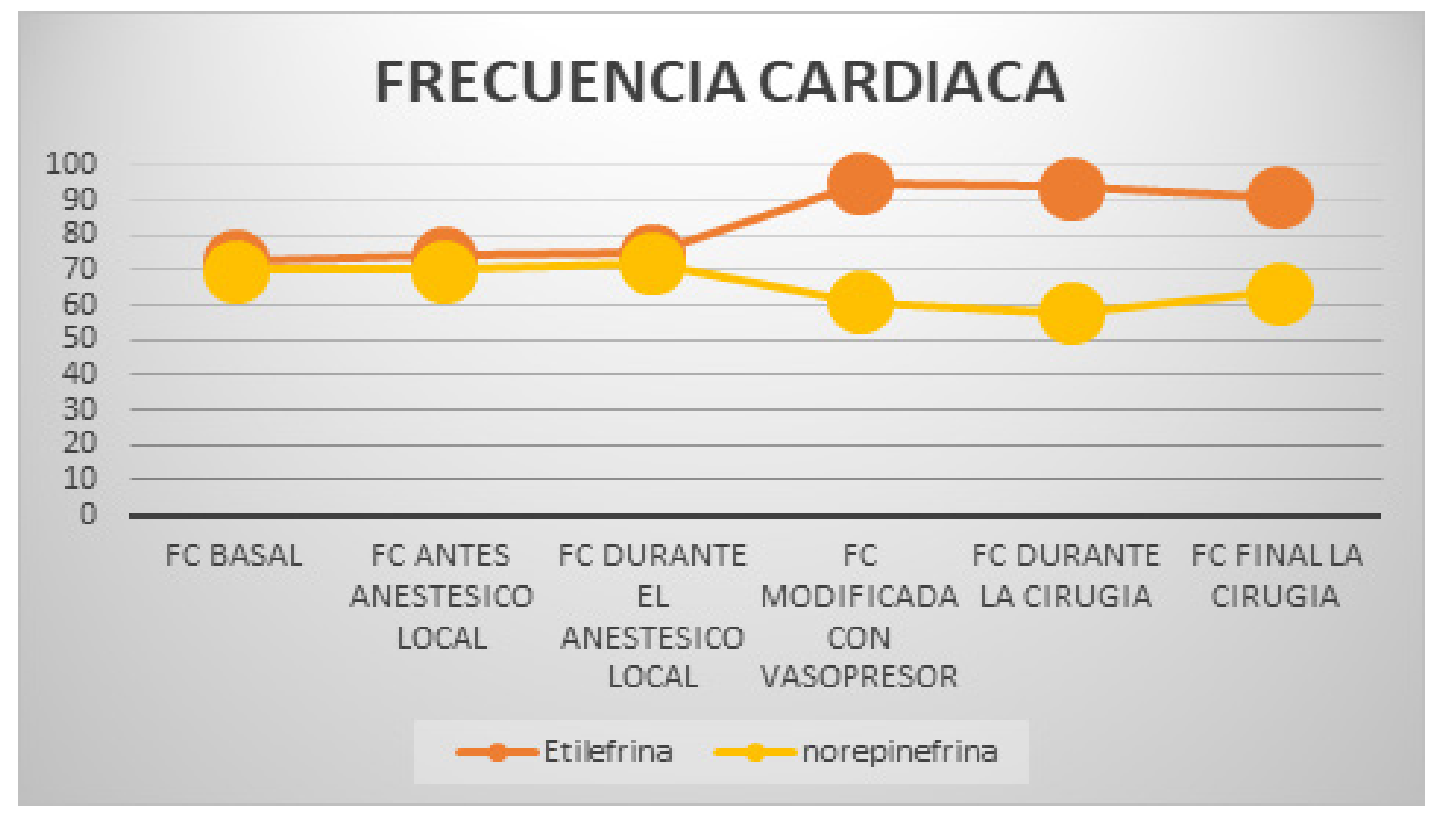

Grafico 4. La frecuencia cardiaca se mantiene estable posterior a la administración de norepinefrina, contrario al uso de Etilefrina, con una tendencia a la taquicardia.

Fuente de Elaboración Propia.

\section{DISCUSIÓN}

En el presente estudio se observa que para prevención de $\mathrm{HIO}$ en artroplastia de cadera se asocia mejor el manejo con noradrenalina, con mínimos cambios en la frecuencia cardiaca que no repercuten hemodinámicamente.

Es importante recalcar que la cantidad de cristaloides fueron mínimas $8-10 \mathrm{ml} / \mathrm{kg}$, excepto en casos que el paciente este hipovolémico, porque el exceso de líquido es causa más frecuente de morbimortalidad perioperatoria y esto puede ser evitable utilizando vasopresores.

Como Robinson y $\mathrm{col}^{5}$ mencionan que la terapia dirigida por objetivo (TDO) se asocia con complicaciones reducidas en la cirugía abdominal mayor, y los estudios sugieren que también hay un papel en la cirugía ortopédica mayor electiva y Habicher - $\mathrm{col}^{11}$ reportan de pacientes sometidos a cirugía de revisión de cadera, demostró una reducción de la morbilidad postoperatoria y la duración de la estadía en pacientes que recibieron TDO. Bengoa y col ${ }^{12}$ en su artículo menciona, como indicador de normovolemia es mantener la diuresis entre 30 y $35 \mathrm{ml} / \mathrm{h}$.

Por otra parte, Errando y col ${ }^{13}$ reporto uso de efedrina como vasopresor para disminuir la aparición de hipotensión arterial. Haberer ${ }^{6}$ menciona que 
la efedrina suele ser menos eficaz y por encima de los $30 \mathrm{mg}$ de efedrina, se recomienda inyectar fenilefrina en bolos de 50 mcg. En el estudio se utilizó noradrenalina con mejor estabilidad hemodinámica a dosis de 0.03 a $0.05 \mathrm{mcg} / \mathrm{kg} / \mathrm{min}$. Hamaji y col ${ }^{14}$ usaron norepinefrina como vasopresor pero $\sin$ mencionar la dosis exacta.

Es importante mantener una presión arterial óptima (presión arterial sistólica por encima 100 mmhg), por su parte Jang y col $^{15}$ concluyen que la duración de la hipotensión intraoperatoria es un factor de riesgo de lesión renal aguda, mientras que Kim y $\operatorname{col}^{16}$ concluyeron que las complicaciones cardiovasculares postoperatorias - un mayor factor de riesgo de mortalidad que se relacionaron con frecuentes episodios de hipotensión intraoperatoria. $\mathrm{Y}$ por último Sessler y $\mathrm{col}^{17}$ demuestran que tener presión arterial media debajo de $60 \mathrm{mmhg}$ está asociado con lesión miocárdica, lesión renal aguda y muerte.

Podemos indicar que el uso de noradrenalina en infusión continua demuestra estabilidad en las presiones arteriales medias durante todo el procedimiento quirúrgico. Así Leblanc y $\mathrm{col}^{18}$ desarrollaron un Software SmarPilot ${ }^{\circledast}$ guiado, que reduce la duración de la hipotensión, las complicaciones postoperatorias y la estancia hospitalaria de los pacientes.

Al usar Etilefrina vemos un ascenso de la frecuencia cardiaca es parte de la advertencia y precauciones del producto, mientras que la norepinefrina demuestra un efecto contrario manteniendo estabilidad en la frecuencia cardiaca al igual que la efedrina como demuestran Meuret y $\mathrm{col}^{19}$ en su estudio.

En el trabajo se encontró que el manejo de noradrenalina es mejor que uso de Etilefrina, sin embargo, Nuñez ${ }^{7}$ en su tesis reporta que la etilefrina es más efectiva que la norepinefrina para el tratamiento de la $\mathrm{HIO}$ en pacientes sanos en edad productiva a quienes se realizaron procedimientos ambulatorios bajo anestesia espinal. Cabe recalcar que usó la norepinefrina en bolos de 16 mcg IV.

Weinstein y $\mathrm{col}^{20}$ reportan que la hipotensión intraoperatoria controlada se ha asociado con beneficios, que incluyen disminución de pérdida de sangre, disminución de transfusión sanguínea y en el caso de las artroplastias mejor implantación con esto mejora las condiciones del campo quirúrgico. Y que para futuras investigaciones y guías clínicas debe existir una diferencia entre el termino de hipotensión inadvertida y controlada.

Conclusión

Podemos concluir que el uso de norepinefrina en infusión continua mantiene estabilidad hemodinámica, tanto en la presión arterial como en la frecuencia cardiaca de los pacientes sometidos a artroplastia total de cadera bajo anestesia raquídea comparada con etilefrina, el uso de etilefrina nos muestra una taquicardia; al empleo de vasopresores disminuyó la cantidad de cristaloides a infundir $y$ evitar periodos prolongados de hipotensión que pueden tener repercusión a nivel renal, cardíaco y la estancia hospitalaria.

No hubo incidentes del uso de vasopresores por vía periférica.

\section{REFERENCIAS}

1. Solans Duran, J. R. Estudio observacional retrospectivo de los bloqueos neuroaxiales en pacientes mayores de 65 años con fractura de extremidad inferior y las complicaciones intraoperatorias derivadas del acto anestésico. Universidad Autónoma de Barcelona. Recercat (Internet). 2013. (Citado el 13 de mayo de 2019); pag:1-50. Disponible en: https://hdl.handle.net/2072/213765

2. Gonzalez H. Protocolo para: estudio clínico de infusión profiláctica de Etilefrina, comparado con placebo, en la prevención del síndrome por implante de cemento óseo en pacientes ASA 1 y 2 sometidos a artroplastia total primaria de cadera bajo anestesia espinal; estudio clínico, aleatorizado, doble ciego. Universidad Nacional de Colombia. Bdigital (Internet).2015 (citado el 12 de junio de 2019); 1-54. Disponible en: http://bdigital.unal.edu.co/50779/

3. Palomino Lourdes, Ramírez Rubén, Vejarano Julio, Ticse Ray. Fractura de cadera en el adulto mayor: la epidemia ignorada en el Perú. Acta méd. peruana [Internet]. 2016 [citado el 20 de julio de 2018];33(1): 15-20. Disponible en: http://www.scielo.org.pe/scielo. php?script $=$ sci_arttext\&pid $=S 1728-59172016000100004 \& \operatorname{lng}=e s$.

4. Zaragoza-lemus, G. Consideraciones anestésicas en cirugía de cadera traumática. Revista Mexicana de Anestesiología (Internet).2017 [Citado el 20 de Julio de 2018]; 40(1), 155-160. Disponible en: https:/www.medigraphic.com/pdfs/rma/cma-2017/cmas171as. $p d f$

5. Robinson, H., Medlock, G., Cranfield, K. Anestesia para cirugía de revisión de cadera. www.wfsahq.org. (Internet). 2017 (Citado el 21 de noviembre 2018); Atow 363. Pag: 1-7. Disponible en: https://www.wfsahq.org/components/com virtual library/media/3a6a9dad8946ef061f805712a39c1930-363-Revision-Hip-Surgery-Spanish.pdf

6. Haberer, J.-P. Anestesia del paciente anciano. EMC - Anestesia-Reanimación (Internet).2014 (citado el 21 de noviembre de 2018); 40(1), 1-18. Disponible en: https://doi.org/10.1016/s12804703(13)66644-x

7. Nuñez H. Evaluación de la efectividad de la etilefrina y de la norepinefrina en el tratamiento de la hipotensión intraoperatoria secundaria a anestesia espinal en el hospital universitario del caribe, cartagena de indias. Repositorio Institucional universidad de Cartagena. (Internet). 2017 (Citado 14 de junio de 2019); pag: 1-31. Disponible en: http://190.242.62.234:8080/jspui/handle/11227/4625

8. Mets B. Should Norepinephrine, Rather than Phenylephrine, 
Be Considered the Primary Vasopresor in Anesthetic Practice?; anesth analg. (Internet).2016 (citado 05 de noviembre de 2017);122 (5):1707-14. Disponible en:https://pdfs.semanticscholarorg/ae99/ c4bf597d42290c93922768fc24e8053a6e4b.pdf

9. Ramos JR. Etilefrina en hipotensión inducida por anestesia raquídea en cesarea. Hospital Maria Auxiliadora, 2015. USMP (internet).2015 (citado el 05 de noviembred de 2017); pag 1- 61. Disponible en:http://www.repositorioacademico.usmp.edu.pe/handle/ usmp/2112

10. Alegre Andrade Patricia, Mamani Ortiz Yercin, Arnica Gamon Alexander. Norepinephrine and Etilefrin as prevention of maternal hipotensión in cesarean section under spinal anesthesia. Rev Cient Cien Mèd [Internet]. 2018 [Citado 28 de Diciembre de 2019]; 21(1):68-77. Disponible en: http://www.scielo.org.bo/scielo. php? script $=$ sci_arttext\&pid $=S 1817-74332018000100008 \& \operatorname{lng}=e n$.

11. Habicher M, Balzer F, Mezger V, et al. Implementation of goal-directed fluid therapy during hip revision arthroplasty: a matched cohort study. Perioperative Medicine. (Internet).2016 [Citado el 10 de Mayo de 2018]; 5:31. Disponible en: https://doi. org/10.1186/s13741-016-0056-x

12. Bengoa F, Carrasco M, Amenábar P, Schweitzer D, Botello E, Klaber I. Optimización perioperatoria del paciente anciano con fractura osteoporótica de cadera. Rev Méd Chile (Internet). 2017[Citado el 10 de Mayo de 2018]; 145(11), 1437-46. Disponible en: https://doi.org/10.4067/s0034-98872017001101437

13. Errando C.L, Peiró C.M, Gimeno A, Soriano J.L. Single shot spinal anesthesia with very low hyperbaric bupivacaine dose (3.75mg) for hip fracture repair surgery in the elderly. A randomized, double blinded study; Rev Esp Anestesiol Reanim (Internet).2014 [Citado el 12 de Junio de 2018]; 61(9), 481-8. Disponible en: $h$ ttps://doi.org/10.1016/j.redar:2014.02.004

14. Hamaji Adilson, Hajjar Ludhmila, Caiero Marcelo, Almeida Juliano, Nakamura Rosana Ely, Osawa Eduardo A et al . Terapia de reposición volémica durante la artroplastia de cadera usando hidroxietilalmidón (130/0,4): comparado con lactato de Ringer reduce la transfusión de sangre alogénica y la infección posoperatoria. Rev. Bras. Anestesiol. [Internet]. 2013 [citado el 16 de febrero de 2019]; 63(1): 36-44. Disponible en: http://www.scielo.br/scielo.php? script $=$ sci arttext\&pid $=$ S0034$70942013000100003 \& \operatorname{lng}=e n$.

15. Jang W. Y, Jung J. K, Lee D. K, Han S. B. Intraoperative hypotension is a risk factor for postoperative acute kidney injury after femoral neck fracture surgery: A retrospective study. BMC Musculoskeletal Disorders. (Internet). 2019 [citado el 13 de junio de 2019]; 20(1), 1-5. Disponible en: https://doi.org/10.1186/s12891-0192496-1

16. Kim B. H, Lee S, Yoo B, Lee W Y, Lim Y, Kim M.C, Kim K. M. Risk factors associated with outcomes of hip fracture surgery in elderly patients. Korean Journal of Anesthesiology. (Internet). 2015 [citado el 12 de Junio de 2019]; 68(6), 561-567. Disponible en: https://doi.org/10.4097/kjae.2015.68.6.561

17. Sessler, D. I., Bloomstone, J. A., Aronson, S., Berry, C., Gan, T. J., Kellum, J. A, et al: Perioperative Quality Initiative consensus statement on intraoperative blood pressure, risk and outcomes for elective surgery. British Journal of Anaesthesia. (Internet).2019 [citado el 12 de Junio de 2019]; 122(5), 563-574. Disponible en: https://doi.org/10.1016/j.bja.2019.01.013

18. LeBlanc, D., Conté, M., Masson, G., Richard, F., Jeanneteau, A., Bouhours, G, et al: SmartPilot ${ }^{\circledR}$ view-guided anaesthesia improves postoperative outcomes in hip fracture surgery: A randomized blinded controlled study. British Journal of Anaesthesia. (Internet).2017 [citado el 12 de Junio de 2019]; 119(5), 1022-1029. Disponible en: $h$ ttps://doi.org/10.1093/bja/aex317

19. Meuret P, Bouvet L, Villet B, Hafez M, Allaouchiche B, Boselli E. Hypobaric unilateral spinal anaesthesia versus general anaesthesia in elderly patients undergoing hip fracture surgical repair: A prospective randomised open trial. Turk J Anaesthesiol Reanim. (Internet).2018 [citado el 12 de Junio de 2019]; 46(2), 121130. Disponible en: https://doi.org/10.5152/TJAR.2018.90699

20. Weinstein S.M, Yadeau J.T, Memtsoudis S.G. Lack of Association between Levels and Length of Intraoperative Controlled Hypotension and Acute Kidney Injury in Total Hip Arthroplasty Patients Receiving Neuraxial Anesthesia. Regional Anesthesia and Pain Medicine. (Internet).2018 [citado el 12 de Junio de 2019]; 43(7), 725-731. Disponible en: https://doi.org/10.1097/ AAP.0000000000000813 